\title{
Distributed control of synchronization of a group of network nodes
}

\author{
Lucia Valentina Gambuzza, Mattia Frasca, Senior, IEEE, Vito Latora
}

\begin{abstract}
Synchronization in networked systems of diffusively coupled oscillators is a ubiquitous, well studied phenomenon, and many techniques have been proposed to control all the network nodes towards a common reference trajectory. In this technical note, instead, we study the problem of synchronizing an arbitrary subset of the nodes of a network at a fixed coupling strength, disregarding the state of the remaining ones. This problem is inspired to what observed in many biological systems, where the functionality of the network is associated to the presence of clusters of synchronous nodes and, on the contrary, is lost when all the nodes synchronize. We propose a distributed control that introduces further interactions between the oscillators guaranteeing the onset and the stability of the desired synchronous manifold. The controllers are designed so that a proper set of symmetries is created in the network. In addition, we show that the stability of the synchronization pattern mainly depends on the degree of the nodes to synchronize and can be, therefore, controlled by increasing it. Finally, we provide numerical examples illustrating our results.
\end{abstract}

Index Terms-Distributed control, complex networks, synchronization.

\section{INTRODUCTION}

In the last few decades, synchronization of networked systems and its control have seen a growing interest in the scientific community due to the theoretical importance of understanding the dynamical behavior of many social, biological and man-made systems, and to their applications to multiagent systems, secure communications, power grids, electronic circuits, and physiological processes [1]. Many efforts have been devoted to explain the mechanisms behind this collective phenomenon and its dependence on the local dynamics of the units, the functional form of the coupling, and the topology of the underlying network [2], [3]. The stability of the synchronous state has been also studied with a wide variety of different approaches, ranging from linearization [4] to Lyapunov functions [3] and contraction theory [5]. At the same time, a number of studies have shed light on the existence of various types of synchronization in networked systems. In fact, beyond the regime where all the network nodes asymptotically tend towards the same trajectory (global synchronization), other scenarios characterized by the appearance of clusters of synchronized nodes [6] or coexistence of synchronized domains and incoherent ones have been predicted theoretically

L. V. Gambuzza and M. Frasca are with the Dipartimento di Ingegneria Elettrica Elettronica e Informatica, University of Catania, Italy. E-mail: [lucia.gambuzza,mattia.frasca]@dieei.unict.it.

V. Latora is with the School of Mathematical Sciences, Queen Mary University of London, London E1 4NS, United Kingdom, and with the Dipartimento di Fisica ed Astronomia, University of Catania and INFN, Italy. Email: v.latora@qmul.ac.uk. and observed experimentally [7]. The latter forms of synchronization are of practical relevance in biological systems (e.g., the phenomenon of unihemispheric sleep [8]) and in manmade systems (e.g., islanding in power grids [9]).

The characterization of the phenomenon of synchronization has prompted the definition of techniques for its control [10], [11]. A particular relevant result in this field refers to the socalled pinning control, which demonstrates that global synchronization can be achieved by introducing feedback actions on a small portion of the network nodes [12]-[17]. Other studies have focused on control techniques aimed at inducing different types of synchronization, as cluster [18]-[20] or chimera states [21]-[23]. The works on cluster synchronization are especially relevant for our study, and, hence, some more details are given below. The aim of controlling cluster synchronization is to induce a synchronization regime where the networked system splits in groups of nodes, also called clusters, such that all the nodes in a cluster are synchronized, while different clusters exhibit distinct dynamical behaviors. This goal is reached with techniques based on pinning control [18], intermittent pinning control [24], aperiodically intermittent pinning control [25], or exploiting the network symmetries [26], [27].

In our technical note we study a slightly different problem: the target is to control the synchronization of an arbitrary subset of the nodes of the networked system, independently of the state of the remaining units. Hence, our aim is not to control the synchronization of all nodes grouped into different clusters as in most of the previously cited works, but of a single cluster of nodes. A similar problem, aiming at stabilizing a single cluster by setting proper interconnection weights and controller gains, has been considered in [26]. The main difference with respect to our work is that in [26] the control action targets at stabilizing a synchronization pattern that already exists, as it is determined by the network structure. In our case we investigate networks of diffusively coupled oscillators (either identical or with heterogeneous dynamics), and propose a distributed control to address the problem; the cluster is created by the controllers which also guarantee its stability. In addition, we assume that the coupling coefficient is not a controllable parameter, but, on the contrary, is fixed.

Our results offer an interesting interpretation. Suppose to consider two identical chaotic systems. As they start from different initial conditions, they will follow distinct evolutions. However, it is well known [28] that they can be synchronized, inducing them to follow exactly the same trajectory, if their interactions are chosen appropriately. For instance, in many cases, one can establish a bidirectional coupling between the two systems and select the proper coupling coefficient so that 
synchronization is achieved. In this work, the perspective is different, as we assume that the two systems are embedded in a network and are not isolated. Quite counter-intuitively, our findings show that inserting a link between the two units is not the most efficient solution to synchronize them, but, on the contrary, it is much more efficient to act on their first neighbors.

\section{Preliminaries}

This section introduces notations and definitions of graph theory [29], [30], group symmetry [31] and algebraic matrices [32], which will be used in the rest of the work.

We indicate by $\mathrm{I}_{n}$ the identity matrix with dimension $n \times n$; with $0_{m \times n}$ a matrix of zeros of dimension $m \times n$ (whenever possible we omit the subscripts in this matrix, even if it is not a square matrix); with $\mathbf{1}_{n}$ the vector of dimension $n$ with unitary elements. A diagonal matrix, say $\mathrm{D}$, with diagonal terms $\lambda_{1}, \ldots, \lambda_{n}$ is indicated as $\mathrm{D}=\operatorname{diag}\left\{\lambda_{1}, \ldots, \lambda_{n}\right\}$.

A graph $\mathcal{G}$ is defined by the set of nodes/vertices $\mathcal{V}(\mathcal{G})=$ $\left\{v_{1}, \ldots, v_{N}\right\}$, and the set of edges/links $\mathcal{E}(\mathcal{G}) \subseteq \mathcal{V}$ x $\mathcal{V}$, whose elements are ordered pairs of distinct vertices. If, for all $\left(v_{i}, v_{j}\right) \in \mathcal{E}(\mathcal{G})$, the edge $\left(v_{j}, v_{i}\right) \in \mathcal{E}(\mathcal{G})$ the graph is undirected, otherwise the graph is direct. In the rest of the work we will consider undirected graphs. To indicate the nodes of $\mathcal{G}$ we will equivalently use $v_{i}$ or, shortly, $i$.

The set of neighbors of node $i$ is denoted by $\mathcal{N}_{i}=\left\{v_{j} \in \mathcal{V}\right.$ : $\left.\left(v_{i}, v_{j}\right) \in \mathcal{E}\right\}$. Let $\mathcal{N}_{i}$ and $\mathcal{N}_{j}$ be the sets of neighbors of node $i$ and node $j$ respectively, the common part or intersection of $\mathcal{N}_{i}$ and $\mathcal{N}_{j}$ is defined as the set of those elements which belong simultaneously to $\mathcal{N}_{i}$ and $\mathcal{N}_{j}$, and indicated as $\mathcal{N}_{i} \cap \mathcal{N}_{j}$. Two sets are disjoint if their intersection is empty. More in general, the intersection of more than two sets, $\mathcal{N}_{i} \cap \mathcal{N}_{j} \cap \ldots \mathcal{N}_{k}$ is the set of nodes which belong simultaneously to $\mathcal{N}_{i}, \mathcal{N}_{j}, \ldots$, and $\mathcal{N}_{k}$. We recall also that the union between two sets $\mathcal{N}_{i}$ and $\mathcal{N}_{j}$ is defined as $\mathcal{N}_{i} \cup \mathcal{N}_{j}=\left\{v_{h} \mid v_{h} \in \mathcal{N}_{i}\right.$ or $\left.v_{h} \in \mathcal{N}_{j}\right\}$ and, analogously, the difference between two sets $\mathcal{N}_{i}$ and $\mathcal{N}_{j}$ is defined as $\mathcal{N}_{i}-\mathcal{N}_{j}=\left\{v_{h} \mid v_{h} \in \mathcal{N}_{i}, v_{h} \notin \mathcal{N}_{j}\right\}$. We indicate the cardinality of a set $\mathcal{N}$, i.e., the number of elements contained in it, as $|\mathcal{N}|$.

Graphs are also represented through their adjacency and Laplacian matrices. The elements of the adjacency matrix A of undirected graphs are defined such that, if there is a link between node $i$ and node $j(i \neq j)$, i.e., if $\left(v_{i}, v_{j}\right) \in \mathcal{E}(\mathcal{G})$, then $a_{i j}=a_{j i}=1$, otherwise $a_{i j}=a_{j i}=0$. We assume that there are no self-loops, i.e., $a_{i i}=0$ for all $i=1, \ldots, N$. We define the degree $k_{i}$ of node $i$ as the number of connections incident on node $i$ : $\sum_{j=1, j \neq i}^{N} a_{i j}=\sum_{j=1, j \neq i}^{N} a_{j i}=k_{i}$, with $i=1,2, \ldots, N$. Given an undirected graph $\mathcal{G}$, the Laplacian matrix $\mathcal{L}(\mathcal{G})$ or, shortly, $\mathcal{L}$, is defined through its coefficients $\mathcal{L}_{i j}=-a_{i j}$ if $i \neq j$, and $\mathcal{L}_{i i}=k_{i}$.

A path between $v_{i} \in \mathcal{V}(\mathcal{G})$ and $v_{j} \in \mathcal{V}(\mathcal{G})$ is a sequence of vertices such that each pair of consecutive vertices in the sequence is connected by an edge. An undirected graph is connected if for each pairs of vertices there exists a path connecting them. The Laplacian $\mathcal{L}(\mathcal{G})$ of a connected, undirected graph is irreducible, symmetric and semi-positive definite, so 0 is an eigenvalue with multiplicity 1 , and all other eigenvalues are strictly positive: $0=\lambda_{1}<\lambda_{2} \leq \lambda_{3} \cdots \leq \lambda_{N}$.
In the following we will also use some tools from group theory which are here briefly recalled. We say that an object has a symmetry if there exists a transformation/operation which, when applied to the object, leaves it unchanged. In particular, for graphs, symmetries are associated to automorphisms, i.e. permutations of the graph nodes that preserve the adjacency of nodes [30]. The symmetries of a graph (more in general of an object) form a mathematical group. Each element of the group is a symmetry of the graph and can be represented as a permutation matrix, $\mathrm{R}_{g}$, i.e., a square matrix containing exactly one non-zero entry for each row and column, with $r_{i j}=1$ if nodes $i$ and $j$ permute, and $r_{i j}=0$ otherwise. The adjacency and the Laplacian matrix are invariant to graph symmetries which implies that $\mathrm{R}_{g} \mathrm{~A}=\mathrm{AR}_{g}$ and $\mathrm{R}_{g} \mathcal{L}=\mathcal{L} \mathrm{R}_{g}$. Furthermore, for any permutation matrix we have that $\mathrm{R}_{g} \mathrm{R}_{g}^{-1}=\mathrm{R}_{g}^{2}=\mathrm{I}_{N}$.

Graph symmetries can be difficult to detect and appropriate tools have been defined [33]. The following Lemma introduces a specific case where a trivial symmetry can be immediately identified.

Lemma 2.1: If two or more nodes $i, j, \ldots, h$ of a graph $\mathcal{G}$, have the same neighborhoods, $\mathcal{N}_{i}=\mathcal{N}_{j}=\ldots=\mathcal{N}_{h}$, then such nodes are symmetric.

Proof: The proof immediately follows from the definition as exchanging any pairs of nodes with the same neighborhood leaves the graph unchanged.

Finally, we recall some properties of the Kronecker product:

$$
\begin{aligned}
(\mathrm{A} \otimes \mathrm{B}) \cdot(\mathrm{C} \otimes \mathrm{D}) & =(\mathrm{A} \cdot \mathrm{C}) \otimes(\mathrm{B} \cdot \mathrm{D}) \\
(\mathrm{A} \otimes \mathrm{B})^{-1} & =\mathrm{A}^{-1} \otimes \mathrm{B}^{-1}
\end{aligned}
$$

with $\mathrm{A}, \mathrm{B}, \mathrm{C}$, and $\mathrm{D}$ matrices of appropriate size.

\section{Problem FORMUlation}

We consider a dynamical network formed by $N$ diffusively coupled identical nodes, with each node being a $n$-dimensional dynamical system:

$$
\dot{\mathbf{x}}_{i}=f\left(\mathbf{x}_{i}\right)+\sigma \sum_{j=1}^{N} a_{i j} \mathrm{H}\left(\mathbf{x}_{j}-\mathbf{x}_{i}\right)
$$

where $\mathbf{x}_{i}=\left(x_{i 1}, x_{i 2}, \ldots, x_{i n}\right)^{T} \in \mathbb{R}^{n}$ is the state variable of node $i, f\left(\mathbf{x}_{i}\right): \mathbb{R}^{n} \rightarrow \mathbb{R}^{n}$ is the local dynamics, the constant $\sigma>0$ represents the coupling strength, and $\mathrm{H} \in \mathbb{R}^{n \times n}$ is a constant matrix of 0-1 coefficients, representing how the coupled variables are linked. Eqs. (3) are commonly used to model the dynamics of various systems of interacting oscillators, from ecological, circadian, electronic and neuronal networks to power grids [2]. Equivalently, the dynamical equations (3) of the network can be rewritten in terms of the graph Laplacian $\mathcal{L}$ as:

$$
\dot{\mathbf{x}}_{i}=f\left(\mathbf{x}_{i}\right)-\sigma \sum_{j=1}^{N} \mathcal{L}_{i j} \mathrm{Hx}_{j}
$$

or, in compact form:

$$
\dot{\mathbf{x}}=F(\mathbf{x})-\sigma \mathcal{L} \otimes \mathrm{H} \cdot \mathbf{x}
$$


where $\mathbf{x}=\left[\mathbf{x}_{1}^{T}, \mathbf{x}_{2}^{T}, \ldots, \mathbf{x}_{N}^{T}\right]^{T}$ is the stack vector of all node states, and $F(\mathbf{x})=\left[f\left(\mathbf{x}_{1}\right)^{T}, f\left(\mathbf{x}_{2}\right)^{T}, \ldots, f\left(\mathbf{x}_{N}\right)^{T}\right]^{T}$ describes the local dynamics of the network.

In the networked dynamical system (4) we introduce distributed controllers $\mathbf{u}_{i}$ with $i=1, \ldots, N$ as follows:

$$
\dot{\mathbf{x}}_{i}=f\left(\mathbf{x}_{i}\right)-\sigma \sum_{j=1}^{N} \mathcal{L}_{i j} \mathrm{Hx}_{j}+\mathbf{u}_{i}
$$

and we assume that they represent further proportional (diffusive) coupling that can be established from node $i$ to the other nodes of the network, i.e.:

$$
\mathbf{u}_{i}=-\sigma \sum_{j=1}^{N} \mathcal{L}^{\prime}{ }_{i j} \mathrm{Hx}_{j}
$$

The controllers act on the dynamical network through the same state variables of the other nodes, and with the same type of coupling (matrix $\mathrm{H}$ ) used for the other links. The coupling coefficient $\sigma$ is fixed to be the same as that in Eq. (6). In this way, Eqs. (7) implement a communication protocol of the same nature as that already used by the networked system to be controlled. The controllers introduce an additional layer of links, modeled through the matrix $\mathcal{L}^{\prime}$, whose elements $\mathcal{L}_{i j}^{\prime}$ with $i \neq j$ can be $-1,1$ or 0 . Namely, $\mathcal{L}_{i j}^{\prime}=-1$ if a link between $i$ and $j$ is added, $\mathcal{L}_{i j}^{\prime}=1$ if an existing link is removed, and $\mathcal{L}_{i j}^{\prime}=0$, otherwise. Finally, the elements $\mathcal{L}_{i i}^{\prime}$ are chosen as $\mathcal{L}_{i i}^{\prime}=-\sum_{j=1, j \neq i}^{N} \mathcal{L}_{i j}^{\prime}$, so that, even if $\mathcal{L}^{\prime}$ is not a Laplacian as it may also contain positive off-diagonal terms, $\mathcal{L}^{\prime}$ is zero row-sum. We indicate as $\mathcal{G}^{\prime \prime}$ the graph formed by the original links and those introduced by the controllers. Links are added or removed so that $\mathcal{L}^{\prime \prime}=\mathcal{L}+\mathcal{L}^{\prime}$ is a Laplacian matrix (in this way $\mathcal{G}^{\prime \prime}$ is unweighted and undirected). Correspondingly, the neighborhood of node $i$ in the graph $\mathcal{G}^{\prime \prime}$ is indicated as $\mathcal{N}_{i}^{\prime \prime}$.

In this work, we study the problem of controlling synchronization of a subset of the network nodes through distributed control. Given a set of $n_{2}<N$ nodes of the network, denoted as $\mathcal{S}_{n_{2}}$, we consider the problem of finding a set of controllers $\mathbf{u}_{i}$ as in Eq. (7) such that the nodes in $\mathcal{S}_{n_{2}}$ are synchronized each other. We indicate with $n_{1}$ the number of remaining nodes, $n_{1}=N-n_{2}$, and relabel the network nodes so that the units to synchronize are indexed as $i=n_{1}+1, \ldots, N$. The aim of our study is, thus, to determine a control such that a synchronous state of the type:

$$
\begin{aligned}
& \mathbf{x}_{1}(t)=\mathbf{s}_{1}(t) \\
& \ldots \\
& \mathbf{x}_{n_{1}}(t)=\mathbf{s}_{n_{1}}(t) \\
& \mathbf{x}_{n_{1}+1}(t)=\mathbf{x}_{n_{1}+2}(t)=\ldots=\mathbf{x}_{N}(t)=\mathbf{s}(t)
\end{aligned}
$$

exists and is exponentially stable. In compact form, we indicate the synchronous state as $\mathbf{x}(t)=\mathbf{x}^{s}(t)=$ $\left[\mathbf{s}_{1}(t)^{T}, \ldots, \mathbf{s}_{n_{1}}(t)^{T}, \mathbf{s}(t)^{T}, \ldots, \mathbf{s}(t)^{T}\right]^{T}$. Notice that, in the most general case, the first $n_{1}$ nodes follow different trajectories, that is $\mathbf{s}_{i}(t) \neq \mathbf{s}_{j}(t)$ for $i, j=1, \ldots, n_{1}$, but eventually some of them may coincide.

\section{MAIN RESUltS}

Our main result is represented by the following theorem.

Theorem 4.1: Consider the dynamical network (6), a set $\mathcal{S}_{n_{2}}$ of $n_{2}$ arbitrary nodes, and a fixed value of the coupling coefficient $\sigma$. If the controllers $\mathbf{u}_{i}$ in Eq. (7) are such that $\mathcal{L}^{\prime \prime}=\mathcal{L}+\mathcal{L}^{\prime}$ fulfils these conditions:

1) $\mathcal{N}_{n_{1}+1}^{\prime \prime}=\ldots=\mathcal{N}_{N}^{\prime \prime}\left(\Rightarrow k_{n_{1}+1}=\ldots=k_{N}\right.$, i.e., the nodes of $\mathcal{S}_{n_{2}}$ have the same degree);

2) the $n_{2}$ nodes in $\mathcal{S}_{n_{2}}$ are not connected each others;

then, a synchronous state $\mathbf{x}^{s}(t)=$ $\left[\mathbf{s}_{1}(t)^{T}, \ldots, \mathbf{s}_{n_{1}}(t)^{T}, \mathbf{s}(t)^{T}, \ldots, \mathbf{s}(t)^{T}\right]^{T}$ exists.

If moreover there exist a diagonal matrix $\mathrm{C}>0$ and two constants $\bar{q}>0$ and $\tau>0$, such that the linear matrix inequality (LMI)

$$
[D f(\mathbf{s}(t))-q \mathrm{H}]^{T} \mathrm{C}+\mathrm{C}[D f(\mathbf{s}(t))-q \mathrm{H}] \leq-\tau \mathrm{I}_{n}
$$

is satisfied for all $q \geq \bar{q}$ and $t>0$ (where $D f(\mathbf{s}(t)$ ) is the Jacobian of $f$ on $\mathbf{s}(t)$ ), and if $\sigma>\bar{q} / n_{1}$ and $\kappa \triangleq k_{n_{1}+1}=$ $\ldots=k_{N}$ is selected so that $\kappa>\frac{\bar{q}}{\sigma}$, then the synchronous state $\mathbf{x}^{s}(t)$ is exponentially stable.

Remark 4.2: As introduced above, the controllers in Theorem 4.1 establish additional links in the network; the resulting network, with Laplacian given by $\mathcal{L}^{\prime \prime}$, is such that the nodes to synchronize have the same sets of neighbors $\mathcal{N}_{i}^{\prime \prime}$ for $i=n_{1}+1, \ldots, N$. Theorem 4.1 requires that the nodes in $\mathcal{S}_{n_{2}}$ have a degree greater than $\bar{q} / \sigma$, a critical value which is larger the smaller is the coupling in the network. If some nodes of $\mathcal{S}_{n_{2}}$, e.g., nodes $\bar{i}$ and $\bar{j}$, are connected in the original graph $\mathcal{G}$, then condition 2) is satisfied by selecting $\mathcal{L}_{\bar{i} \bar{j}}^{\prime}=1$.

Remark 4.3: Let us exemplify the application of Theorem 4.1 for $n_{2}=2$. Let us indicate the two nodes to synchronize as node $\mathrm{A}$ and node $\mathrm{B}$. If $\left|\mathcal{N}_{A} \cup \mathcal{N}_{B}\right|<\bar{q} / \sigma$, we define a set of arbitrary nodes of the network, indicated as $\mathcal{V}_{2} \subseteq\left(\mathcal{V}(\mathcal{G})-\mathcal{N}_{A}-\mathcal{N}_{B}-\{A, B\}\right)$, such that $\left|\mathcal{N}_{A} \cup \mathcal{N}_{B}\right|+\left|\mathcal{V}_{2}\right|>\bar{q} / \sigma$, and select the controller as:

$$
\begin{aligned}
& \mathbf{u}_{A}=\sigma \sum_{j \in\left(\mathcal{N}_{B}-\mathcal{N}_{A}\right)} \mathrm{H}\left(\mathbf{x}_{j}-\mathbf{x}_{A}\right)+\sigma \sum_{j \in \mathcal{V}_{2}} \mathrm{H}\left(\mathbf{x}_{j}-\mathbf{x}_{A}\right) \\
& \mathbf{u}_{B}=\sigma \sum_{j \in\left(\mathcal{N}_{A}-\mathcal{N}_{B}\right)} \mathrm{H}\left(\mathbf{x}_{j}-\mathbf{x}_{B}\right)+\sigma \sum_{j \in \mathcal{V}_{2}} \mathrm{H}\left(\mathbf{x}_{j}-\mathbf{x}_{B}\right)
\end{aligned}
$$

In the case that $\left|\mathcal{N}_{A} \cup \mathcal{N}_{B}\right|>\bar{q} / \sigma$, then $\mathcal{V}_{2}=\emptyset$, and the last term in the right hand side of each Eq. (10) vanishes.

In addition, as the links are bidirectional, complementary control actions are set to the other end-nodes of the links.

Proof of Theorem 4.1: Existence of the synchronous solution. The application of the controllers (7) in the dynamical network (6) leads to:

$$
\dot{\mathbf{x}}_{i}=f\left(\mathbf{x}_{i}\right)-\sigma \sum_{j=1}^{N} \mathcal{L}_{i j}^{\prime \prime} \mathrm{H} \mathbf{x}_{j}
$$

or, in compact form

$$
\dot{\mathbf{x}}=F(\mathbf{x})-\sigma \mathcal{L}^{\prime \prime} \otimes \mathrm{H} \cdot \mathbf{x}
$$


System (12) represents a dynamical network of units coupled through the Laplacian $\mathcal{L}^{\prime \prime}$. $\mathcal{L}^{\prime \prime}$ satisfies conditions 1)2 ), which guarantee for Lemma 2.1 that the $n_{2}$ nodes are symmetric. This means that the equations of motion of the dynamical network remain unchanged if any of the nodes of $\mathcal{S}_{n_{2}}$ is permuted with any other node of $\mathcal{S}_{n_{2}}$. The nodes of $\mathcal{S}_{n_{2}}$ have, thus, the same equations of motions. If these nodes have the same initial conditions, they remain synchronized at any time. Hence, system (12) admits an invariant synchronization manifold and, so, a solution $\mathrm{x}^{s}$ exists.

Stability of the synchronous solution. It remains to demonstrate that this solution is exponentially stable. To this aim, we first demonstrate that the synchronous state $\mathbf{x}^{s}(t)$ of the dynamical network (11) is exponentially stable, if

$$
\dot{\zeta}=(D f-\sigma \kappa \mathrm{H}) \zeta
$$

is exponentially stable, where $\zeta \in \mathbb{R}^{n}$ and $\kappa$ is the degree that the nodes to synchronize have in $\mathcal{G}^{\prime \prime}$.

We consider the synchronous state $\mathbf{x}^{s}$ and define $\mathbf{x}=\mathbf{x}^{s}+\eta$, that is, $\mathbf{x}_{i}=\mathbf{x}_{i}^{s}+\eta_{i}$, with $\eta=\left[\begin{array}{llll}\eta_{1}^{T} & \eta_{2}^{T} & \ldots & \eta_{N}^{T}\end{array}\right]^{T}$, and $\eta_{i} \in \mathbb{R}^{n}, i=1, \ldots, N$.

We proceed by linearizing Eqs. (12) around $\mathbf{x}^{s}$ :

$$
\dot{\eta}=D F \cdot \eta-\sigma\left(\mathcal{L}^{\prime \prime} \otimes \mathrm{H}\right) \cdot \eta
$$

with $D F=\operatorname{diag}\left\{D f_{1}, D f_{2}, \ldots, D f_{n_{1}}, D f_{s}, \ldots, D f_{s}\right\}$, where $D f_{1}=D f\left(\mathbf{s}_{1}(t)\right), D f_{2}=D f\left(\mathbf{s}_{2}(t)\right), \ldots, D f_{n_{1}}=$ $D f\left(\mathbf{s}_{n_{1}}(t)\right)$, and $D f_{s}=D f(\mathbf{s}(t))$. Note that the structure of this matrix with the first $n_{1}$ blocks being different and the other $n_{2}$ blocks being identical derives from the choice of the synchronous state $\mathbf{x}^{s}$ as in (8).

We now aim at defining proper transformed variables leading to a system of equations that can be decoupled. To do this, we consider the following matrix $\mathrm{R} \in \mathbb{R}^{n \times n}$

$$
\mathrm{R}=\left[\begin{array}{cc}
\mathrm{I}_{n_{1}} & 0 \\
0 & \mathbf{1}_{n_{2}} \cdot \mathbf{1}_{n_{2}}^{T}-\mathrm{I}_{n_{2}}
\end{array}\right]
$$

and note that this matrix is symmetric and, thus, for the Schur's lemma, there exists an orthogonal matrix $M$ such that $\mathrm{M}^{-1} \mathrm{RM}=\operatorname{diag}\left\{\lambda_{1}(\mathrm{R}), \ldots, \lambda_{N}(\mathrm{R})\right\}$, where $\lambda_{1}(\mathrm{R}), \ldots, \lambda_{N}(\mathrm{R})$, are the eigenvalues of $\mathrm{R}$. These eigenvalues are known and can be ordered in the following way: $\lambda_{1}(\mathrm{R})=\ldots=\lambda_{n_{1}}(\mathrm{R})=1, \lambda_{n_{1}+1}(\mathrm{R})=n_{2}-1$, $\lambda_{n_{1}+2}(\mathrm{R})=\ldots=\lambda_{N}(\mathrm{R})=-1$ [29]. For $n_{2}=2, \mathrm{R}$ is the permutation matrix associated to the symmetry in $\mathcal{L}^{\prime \prime}$ of the two nodes to synchronize. In the more general case, $n_{2}>2$, $\mathrm{R}$ is no more a permutation matrix as $\mathrm{R}^{2} \neq \mathrm{I}_{N}$.

It is known that any permutation matrix $\mathrm{R}_{g}$ of a symmetry of a network with Laplacian $\mathcal{L}$ has the property that, given $M$ such that $\mathrm{M}^{-1} \mathrm{R}_{g} \mathrm{M}$ is diagonal, then $\mathrm{M}^{-1} \mathcal{L} \mathrm{M}$ is blockdiagonal [34]. In the following, we prove and use the fact that this property also applies with $\mathrm{R}$ as in Eq. (15).

We use $\mathrm{M}$, which diagonalizes $\mathrm{R}$, to define the following transformed variables

$$
\xi=\left(\mathrm{M}^{-1} \otimes \mathrm{I}_{n}\right) \cdot \eta
$$

By substituting the new variables into Eq. (14) and applying the properties of the Kronecker product (1), we obtain

$$
\begin{aligned}
& \dot{\xi}=\left(\mathrm{M}^{-1} \otimes \mathrm{I}_{n}\right) D F\left(\mathrm{M}^{-1} \otimes \mathrm{I}_{n}\right)^{-1} \xi- \\
& -\sigma\left(\mathrm{M}^{-1} \otimes \mathrm{I}_{n}\right)\left(\mathcal{L}^{\prime \prime} \otimes \mathrm{H}\right)\left(\mathrm{M}^{-1} \otimes \mathrm{I}_{n}\right)^{-1} \xi= \\
& =\left(\mathrm{M}^{-1} \otimes \mathrm{I}_{n}\right) D F\left(\mathrm{M} \otimes \mathrm{I}_{n}\right) \xi-\sigma\left(\mathrm{M}^{-1} \mathcal{L}^{\prime \prime} \mathrm{M}\right) \otimes \mathrm{H} \xi
\end{aligned}
$$

In what follows we make explicit the two terms appearing in Eq. (17). We first derive the term $\left(\mathrm{M}^{-1} \otimes \mathrm{I}_{n}\right) \cdot D F \cdot\left(\mathrm{M} \otimes \mathrm{I}_{n}\right)$. To this aim, let us write $\mathrm{M}$ as

$$
\mathrm{M}=\left[\begin{array}{cc}
\mathrm{I}_{n_{1}} & 0 \\
0^{T} & \mathrm{~V}_{n 2}
\end{array}\right]
$$

where $\mathrm{V}_{n_{2}}$ is an orthogonal matrix such that $\mathrm{V}_{n 2}^{T}\left(\mathbf{1}_{n_{2}} \cdot \mathbf{1}_{n_{2}}^{T}-\right.$ $\left.\mathrm{I}_{n_{2}}\right) \mathrm{V}_{n_{2}}=\operatorname{diag}\left\{\lambda_{n_{1}+1}(\mathrm{R}), \ldots, \lambda_{N}(\mathrm{R})\right\}$, and, correspondingly, let us partition $D F$ as

$$
D F=\left[\begin{array}{cc}
D F_{11} & 0 \\
0 & D F_{22}
\end{array}\right]
$$

with $D F_{11} \in \mathbb{R}^{\left(n_{1} \cdot n\right) \times\left(n_{1} \cdot n\right)}$ and $D F_{22} \in \mathbb{R}^{\left(n_{2} \cdot n\right) \times\left(n_{2} \cdot n\right)}$.

We get

$$
\begin{aligned}
& \left(\mathrm{M}^{-1} \otimes \mathrm{I}_{n}\right) \cdot D F \cdot\left(\mathrm{M} \otimes \mathrm{I}_{n}\right)= \\
& =\left[\begin{array}{cc}
\mathrm{I}_{n_{1} \cdot n} & 0 \\
0^{T} & \mathrm{~V}_{n_{2}}^{T} \otimes \mathrm{I}_{n}
\end{array}\right] \cdot\left[\begin{array}{cc}
D F_{11} & 0 \\
0 & D F_{22}
\end{array}\right] \cdot\left[\begin{array}{cc}
\mathrm{I}_{n_{1} \cdot n} & 0 \\
0^{T} & \mathrm{~V}_{n_{2}} \otimes \mathrm{I}_{n}
\end{array}\right]= \\
& =\left[\begin{array}{cc}
D F_{11} & \\
0^{T} & \left(\mathrm{~V}_{n_{2}}^{T} \otimes \mathrm{I}_{n}\right) \cdot D F_{22} \cdot\left(\mathrm{V}_{n_{2}} \otimes \mathrm{I}_{n}\right)
\end{array}\right]= \\
& =\left[\begin{array}{cc}
D F_{11} & 0 \\
0^{T} & \mathrm{I}_{n_{2}} \otimes D f_{s}
\end{array}\right]
\end{aligned}
$$

where we have used that:

$$
\mathrm{M} \otimes \mathrm{I}_{n}=\left[\begin{array}{cc}
\mathrm{I}_{n_{1}} & 0 \\
0^{T} & \mathrm{~V}_{n_{2}}
\end{array}\right] \otimes \mathrm{I}_{n}=\left[\begin{array}{cc}
\mathrm{I}_{n_{1}} \otimes \mathrm{I}_{n} & 0 \\
0^{T} & \mathrm{~V}_{n_{2}} \otimes \mathrm{I}_{n}
\end{array}\right]
$$

Hence, we obtain that

$$
\left(\mathrm{M}^{-1} \otimes \mathrm{I}_{n}\right) \cdot D F \cdot\left(\mathrm{M} \otimes \mathrm{I}_{n}\right)=D F
$$

We now compute the term $\left(\mathrm{M}^{-1} \mathcal{L}^{\prime \prime} \mathrm{M}\right)$ appearing in Eqs. (17). To this aim, we write the Laplacian matrix $\mathcal{L}^{\prime \prime}$ as

$$
\mathcal{L}^{\prime \prime}=\left[\begin{array}{ll}
\mathcal{L}_{11}^{\prime \prime} & \mathcal{L}_{12}^{\prime \prime} \\
\mathcal{L}_{21}^{\prime \prime} & \mathcal{L}_{22}^{\prime \prime}
\end{array}\right]
$$

where the blocks $\mathcal{L}_{11}^{\prime \prime}$ and $\mathcal{L}_{22}^{\prime \prime}$ have size $n_{1} \times n_{1}$ and $n_{2} \times n_{2}$, respectively. Furthermore, $\mathcal{L}_{21}^{\prime \prime}=\mathcal{L}_{12}^{\prime \prime} T$ with size $n_{2} \times n_{1}$.

We obtain:

$$
\begin{aligned}
& \left(\mathrm{M}^{-1} \mathcal{L}^{\prime \prime} \mathrm{M}\right)= \\
& =\left[\begin{array}{cc}
\mathrm{I}_{n_{1}} & 0 \\
0^{T} & \mathrm{~V}_{n_{2}}^{T}
\end{array}\right] \cdot\left[\begin{array}{cc}
\mathcal{L}_{11}^{\prime \prime} & \mathcal{L}_{12}^{\prime \prime} \\
\mathcal{L}_{12}^{\prime \prime T} & \mathcal{L}_{22}^{\prime \prime}
\end{array}\right] \cdot\left[\begin{array}{cc}
\mathrm{I}_{n_{1}} & 0 \\
0^{T} & \mathrm{~V}_{n_{2}}
\end{array}\right]= \\
& =\left[\begin{array}{cc}
\mathcal{L}_{11}^{\prime \prime} & \mathcal{L}_{12}^{\prime \prime} \mathrm{V}_{n_{2}} \\
\mathrm{~V}_{n_{2}}^{T} \mathcal{L}_{12}^{\prime \prime T} & \mathrm{~V}_{n_{2}}^{T} \mathcal{L}_{22}^{\prime \prime} \mathrm{V}_{n_{2}}
\end{array}\right]
\end{aligned}
$$

Since $\mathcal{L}^{\prime}$ is chosen so that the nodes $n_{1}+1, \ldots, N$ are disconnected (condition 2) of Theorem 4.1), then $\mathcal{L}_{22}^{\prime \prime}=$ $\operatorname{diag}\{\kappa, \ldots, \kappa\}$. It follows that $\mathrm{V}_{n_{2}}^{T} \mathcal{L}_{22}^{\prime \prime} \mathrm{V}_{n_{2}}=\kappa \mathrm{V}_{n_{2}}^{T} \mathrm{~V}_{n_{2}}=$ $\kappa \mathrm{I}_{n_{2}}$. 
The term $\mathcal{L}_{12}^{\prime \prime}$ also has a particular structure. This term, in fact, represents how the nodes $n_{1}+1, \ldots, N$ are connected to the rest of the network. Since, for condition 1) of Theorem 4.1, they have the same sets of neighbors, then each row $i$ of $\mathcal{L}_{12}^{\prime \prime}$ contains either all ones (if node $i$ is connected to the nodes $n_{1}+1, \ldots, n_{2}$ ) or all zeros (if it is not connected), that is:

$$
\mathcal{L}_{12}^{\prime \prime}=\left[\begin{array}{ccc}
0 & \ldots & 0 \\
\vdots & & \vdots \\
1 & \ldots & 1 \\
\vdots & & \vdots \\
0 & \ldots & 0
\end{array}\right]
$$

Now, consider that $\mathrm{V}_{n_{2}}$ is formed by $n_{2}$ orthonormal eigenvectors of the matrix $\mathbf{1}_{n_{2}} \cdot \mathbf{1}_{n_{2}}^{T}-\mathrm{I}_{n_{2}}$, i.e., $\mathrm{V}_{n_{2}}=$ $\left[\begin{array}{llll}\mathbf{V}_{1} & \mathbf{V}_{2} & \ldots & \mathbf{V}_{n_{2}}\end{array}\right]$. In addition, $\mathbf{V}_{1}=\left[\frac{1}{\sqrt{n}} \ldots \frac{1}{\sqrt{n}}\right]^{T}$ and, thus, $\mathbf{1}_{n_{2}}^{T} \cdot \mathbf{V}_{1}=\frac{N}{\sqrt{N}}$, and, since $\mathbf{1}_{n_{2}}^{T}$ is orthogonal to $\mathbf{V}_{2}, \ldots, \mathbf{V}_{n_{2}}$, we have that $\mathbf{1}_{n_{2}}^{T} \cdot \mathbf{V}_{2}=0, \ldots, \mathbf{1}_{n_{2}}^{T} \cdot \mathbf{V}_{n_{2}}=0$. This yields:

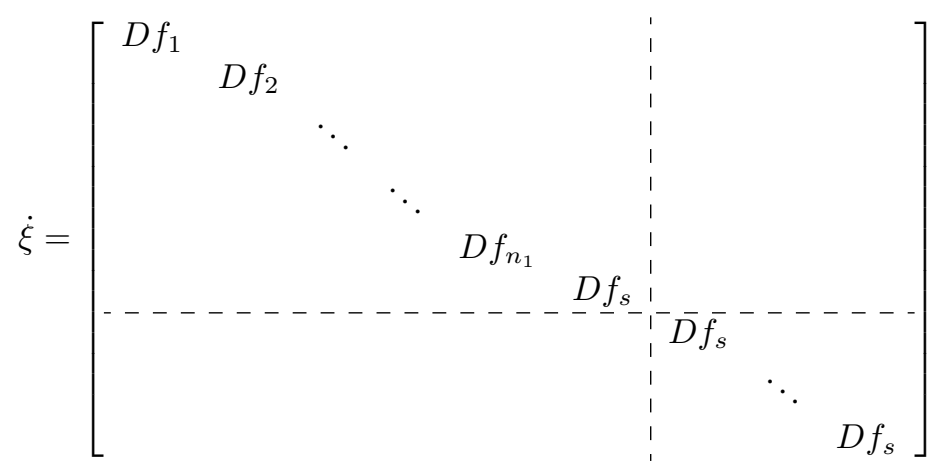

$$
\mathcal{L}_{12}^{\prime \prime} \cdot \mathrm{V}_{n_{2}}=\left[\begin{array}{cccc}
0 & 0 & \ldots & 0 \\
\vdots & & & \\
\frac{N}{\sqrt{N}} & 0 & \ldots & 0 \\
\vdots & & & \\
0 & 0 & \ldots & 0
\end{array}\right]
$$

In conclusion, we get:

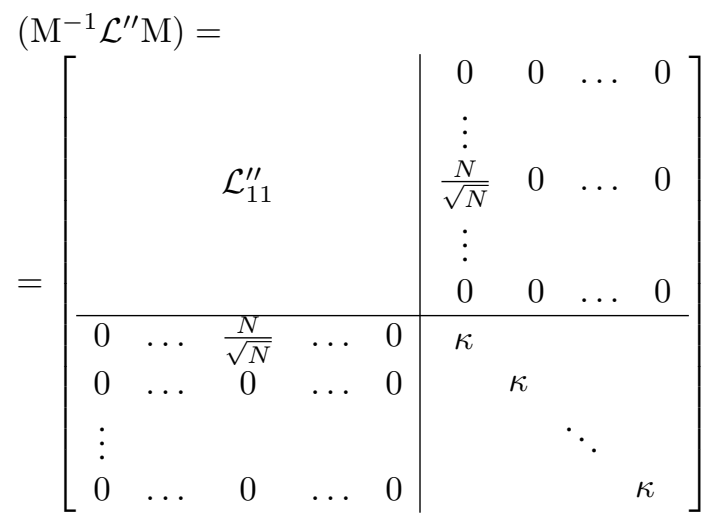

Substituting Eqs. (19) and (24) into Eq. (17), we obtain:

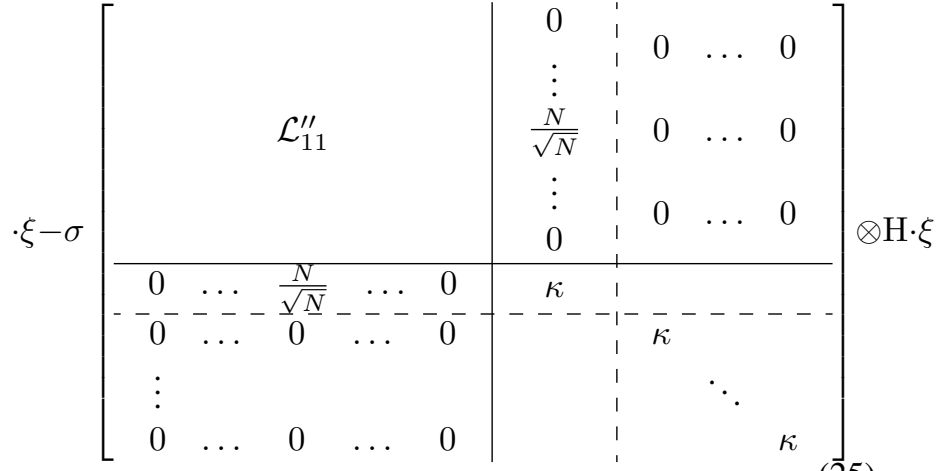

The Laplacian matrix appearing in Eqs. (25) is divided by the continuous lines in four submatrices of size $n_{1} \times n_{1}, n_{1} \times$ $n_{2}, n_{2} \times n_{1}$, and $n_{2} \times n_{2}$. The zeros appearing in the two offdiagonal submatrices suggest another subdivision, emphasized by dashed lines, into blocks of size $\left(n_{1}+1\right) \times\left(n_{1}+1\right),\left(n_{1}+\right.$ $1) \times\left(n_{2}-1\right),\left(n_{2}-1\right) \times\left(n_{1}+1\right)$, and $\left(n_{2}-1\right) \times\left(n_{2}-1\right)$. Hence Eqs. (25) split into a block of $n_{1}+1$ coupled $n$-dimensional linear time-varying systems and $n_{2}-1$ decoupled, identical blocks of $n$-dimensional linear time-varying systems of the form:

$$
\dot{\zeta}=(D f-\sigma \kappa \mathrm{H}) \zeta
$$

The first block of Eqs. (25) describes the dynamics within the synchronization manifold; variations within this manifold do not affect the stability of the synchronous state as the manifold is invariant [35] (for the equivalence between transverse exponential stability of an invariant manifold and exponential stability of the transverse linearized system see also [36]). The other $n_{2}-1$ blocks represent the modes transverse to this manifold. The synchronous state is, therefore, exponentially stable if Eqs. (26) are exponentially stable.

To prove the exponentially stability of Eqs. (26), consider that, since $\sigma>\bar{q} / n_{1}$, we can select $\mathcal{L}^{\prime}$ such that $\left|\mathcal{N}_{n_{1}+1}\right|=$ $\ldots=\left|\mathcal{N}_{n_{2}}\right|=\kappa$ is greater than $\bar{q} / \sigma$. This yields $\sigma \kappa>\bar{q}$. From condition 9 , by using the Lyapunov function $V=\eta^{T} \mathrm{C} \eta$, the exponential stability of Eqs. (26) follows.

Remark 4.4: We note that the conditions on $\mathcal{L}^{\prime \prime}$ in Theorem 4.1 are mild. In particular, the results highlight that to synchronize the nodes in $\mathcal{S}_{n_{2}}$ it is required that each of them has at least $\kappa$ links with the other network nodes. The theorem states that it is not important to which nodes they are actually connected, but only their number.

Remark 4.5: To check the condition for the stability in Theorem 4.1, one can numerically simulate a trajectory of system (3) subjected to the constraints of the synchronous state, i.e., $\mathbf{x}_{n_{1}+1}(t)=\mathbf{x}_{n_{1}+2}(t)=\ldots=\mathbf{x}_{N}(t)$, obtain $\mathbf{s}(t)=\mathbf{x}_{n_{1}+1}(t)$ 
and numerically check LMI (9) with this trajectory. If the trajectory is sufficiently long, then one can assume that the behavior is representative for the whole chaotic attractor. The condition expressed by this hypothesis essentially establishes that the units of the dynamical network are such that they can be fully synchronized for a large coupling coefficient, where the value of the threshold for synchronization is derived from the application of the LMI condition. This assumption is also used in [37], [38]. For proper matrices H, many systems, e.g., Chua's circuit, Rössler system, Lorenz system, and many others, satisfy the property that synchronization is ensured for a coupling coefficient larger than a threshold. Note that the existence of a threshold for synchronization can be also established with other techniques [4], and Theorem 4.1 also applies in these cases.

Finally, we present the extension of Theorem 4.1 to the case of dynamical networks of nodes with heterogeneous dynamics.

Theorem 4.6: Consider the following dynamical network

$$
\dot{\mathbf{x}}_{i}=f_{i}\left(\mathbf{x}_{i}\right)-\sigma \sum_{j=1}^{N} \mathcal{L}_{i j} \mathrm{Hx}_{j}+\mathbf{u}_{i}
$$

a set $\mathcal{S}_{n_{2}}$ of $n_{2}$ arbitrary nodes with identical dynamics, i.e., $f_{i}\left(\mathbf{x}_{i}\right)=f\left(\mathbf{x}_{i}\right)$ for all $i \in \mathcal{S}_{n_{2}}$, and a fixed value of the coupling coefficient $\sigma$. If the controllers $\mathbf{u}_{i}$ in Eq. (7), are such that $\mathcal{L}^{\prime \prime}=\mathcal{L}+\mathcal{L}^{\prime}$ fulfils these conditions:

1) $\mathcal{N}_{n_{1}+1}^{\prime \prime}=\ldots=\mathcal{N}_{N}^{\prime \prime}\left(\Rightarrow k_{n_{1}+1}=\ldots=k_{N}\right.$, i.e., the nodes of $\mathcal{S}_{n_{2}}$ have the same degree);

2) the $n_{2}$ nodes in $\mathcal{S}_{n_{2}}$ are not connected each others;

then a synchronous state $\mathbf{x}^{s}=$ $\left[\mathbf{s}_{1}(t)^{T}, \ldots, \mathbf{s}_{n_{1}}(t)^{T}, \mathbf{s}(t)^{T}, \ldots, \mathbf{s}(t)^{T}\right]^{T}$ exists.

If moreover there exist a diagonal matrix $\mathrm{C}>0$ and two constants $\bar{q}>0$ and $\tau>0$, such that the LMI

$$
[D f(\mathbf{s}(t))-q \mathrm{H}]^{T} \mathrm{C}+\mathrm{C}[D f(\mathbf{s}(t))-q \mathrm{H}] \leq-\tau \mathrm{I}_{n}
$$

is satisfied for all $q \geq \bar{q}$ and $t>0$ (where $D f(\mathbf{s}(t)$ ) is the Jacobian of $f$ on $\mathbf{s}(t)$ ), and if $\sigma>\bar{q} / n_{1}$ and $\kappa \triangleq k_{n_{1}+1}=$ $\ldots=k_{N}$ is selected such that $\kappa>\frac{\bar{q}}{\sigma}$, then the synchronous state $\mathbf{x}^{s}(t)$ is exponentially stable.

Proof: The proof is similar to that of Theorem 4.1 by replacing $D f_{1}=D f_{1}\left(\mathbf{s}_{1}(t)\right), D f_{2}=D f_{2}\left(\mathbf{s}_{2}(t)\right), \ldots, D f_{n_{1}}=$ $D f_{n_{1}}\left(\mathbf{s}_{n_{1}}(t)\right)$, in the definition of $D F$.

\section{EXAMPLES}

We illustrate our control technique with two examples.

Example 1. In the first example, we consider the case where the dynamical systems at the network nodes are identical Chua's oscillators [39]. The state of each node is then described by a three-dimensional vector $\left(x_{1}, x_{2}, x_{3}\right)$ whose dynamics can be written (in a dimensionless form) as:

$$
\begin{aligned}
& \dot{x}_{1}=\alpha\left(x_{2}-x_{1}+g\left(x_{1}\right)\right) \\
& \dot{x}_{2}=x_{1}-x_{2}+x_{3} \\
& \dot{x}_{3}=-\beta x_{2}-\gamma x_{3}
\end{aligned}
$$

where $g\left(x_{1}\right)$ is the piecewise linear function defined as $g\left(x_{1}\right)=-b x_{1}+0.5(b-a)\left(\left|x_{1}+1\right|-\left|x_{1}-1\right|\right)$ with $a=-1.27$

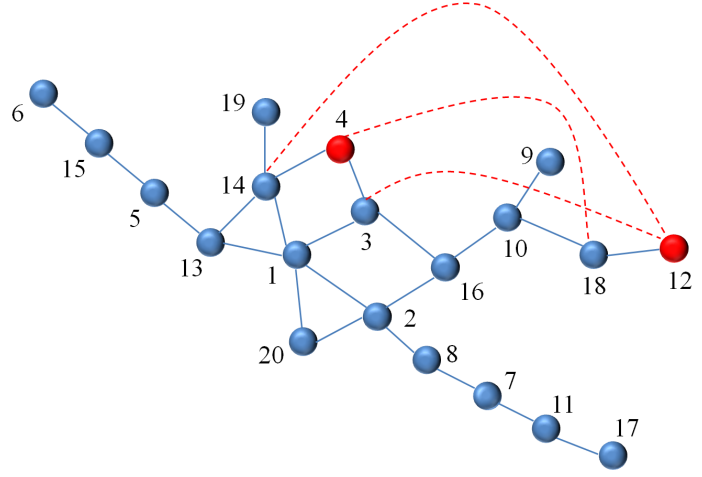

Fig. 1. Study case of a network with $N=20$ nodes and $L=23$ edges (blue, continuous lines). Each of the nodes is a Chua's oscillator and we assume that we want to synchronize the two nodes 4 and 12 highlighted in red. The obtained control scheme is obtained by the creation of an additional layer of links, reported with red dashed lines.

and $b=-0.68$. The other parameters of the Chua's oscillator are chosen so that the system is chaotic, i.e., $\alpha=10, \beta=15$ and $\gamma=0.0385$.

We consider a dynamical network (6) of $N=20$ Chua's oscillators coupled as in Fig. 1, where the original links of the network are shown in solid lines. As for the functional form of the coupling, we assume that the interaction between two Chua's oscillators is realized through the first and the second state variables. This corresponds to adopting a coupling matrix $\mathrm{H}$ of the form $\mathrm{H}=\operatorname{diag}\{1,1,0\}$. We also fix the coupling coefficient to the value $\sigma=2$, and we suppose that the nodes we want to synchronize are nodes 4 and 12 , i.e., $n_{2}=2$ and $\mathcal{S}_{n_{2}}=\{4,12\}$.

To analyze the temporal evolution of the network, we evaluate the global synchronization error $e(t)=$ $\sqrt{\frac{1}{N^{2}} \sum_{i, j}\left\|\mathbf{x}_{i}(t)-\mathbf{x}_{j}(t)\right\|^{2}}$, which takes into account all the possible pairs of nodes of the network, and the synchronization error between the nodes in $\mathcal{S}_{n_{2}}=\{4,12\}$ as $e_{4,12}(t)=\left\|\mathbf{x}_{4}(t)-\mathbf{x}_{12}(t)\right\|$.

For $\sigma=2$ the dynamical network is not synchronized as the trend of $e(t)$, reported in Fig. 2(a), clearly indicates. Correspondingly, also the two nodes in $\mathcal{S}_{n_{2}}$ are not synchronized as shown by $e_{4,12}(t)$ in Fig. 2(b).

We now apply the control technique of Theorem 4.1. The dynamical network of Chua's oscillators satisfies LMI (9) with $\bar{q}=4.5$, hence $\frac{\bar{q}}{\sigma}=2.25$. By setting $\kappa=3$, we have $\sigma \kappa>$ $\bar{q}$, that is, the condition for the exponential stability of the synchronous state is satisfied.

It remains to select the links in $\mathcal{L}^{\prime}$ such that the neighborhoods of nodes 4 and 12 in $\mathcal{L}^{\prime \prime}$ are equal and have cardinality 3 . In the original network, the node 4 is linked to nodes 3 and 14 , i.e., $\mathcal{N}_{4}=\{3,14\}$, while node 12 is linked to node 18 , i.e., $\mathcal{N}_{12}=\{18\}$. We then select $\mathcal{L}^{\prime}$ by considering the following links: $(4,18),(12,14)$, and $(12,3)$. These are the links highlighted as dashed lines in Fig. 1. This yields to the same set of neighbors for the two nodes 4 and 12 and to the same degree, equal to $\kappa=3$. Accordingly the controllers for the nodes 4 and 12 are: 


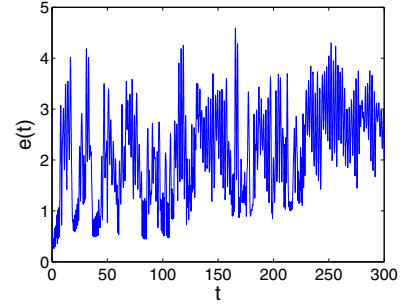

(a)

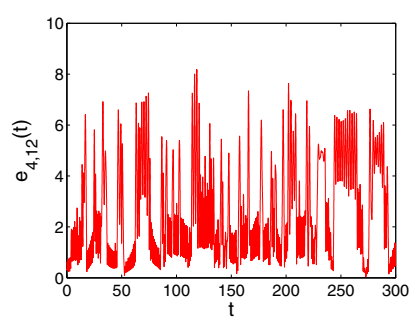

(b)
Fig. 2. Behavior of the dynamical network of Fig. 1 without control: (a) global synchronization error $e(t)$; (b) synchronization error $e_{4,12}(t)$ between nodes 4 and 12 . Without control the whole network is not synchronized.

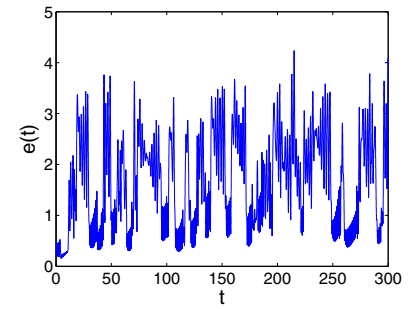

(a)

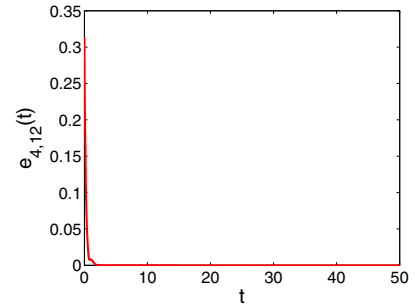

(b)
Fig. 3. Behavior of the dynamical network of Fig. 1 in presence of control (30): (a) global synchronization error $e(t)$; (b) synchronization error $e_{4,12}(t)$ between nodes 4 and 12. Control enables synchronization between the two target nodes, 4 and 12, while the rest of the network remains not synchronized.

$$
\begin{aligned}
& u_{4}=\sigma \sum_{j \in\left(\mathcal{N}_{12}-\mathcal{N}_{4}\right)=\{18\}} \mathrm{H}\left(\mathbf{x}_{j}-\mathbf{x}_{4}\right) \\
& u_{12}=\sigma \sum_{j \in\left(\mathcal{N}_{4}-\mathcal{N}_{12}\right)=\{3,14\}} \mathrm{H}\left(\mathbf{x}_{j}-\mathbf{x}_{12}\right)
\end{aligned}
$$

In addition, as the links are bidirectional, complementary control actions are set to the other end-nodes of the links, i.e., nodes 3,14 , and 18. Fig. 3 shows that by applying the controllers specified above the nodes 4 and 12 are synchronized, while the rest of the network is not. Although it is not the aim of this work to inspect the whole network behavior, we notice that the common neighbors of the two nodes 4 and 12 to be synchronized, i.e., nodes in $\mathcal{N}_{4}^{\prime}=\mathcal{N}_{12}^{\prime}=\{3,14,18\}$, display some correlation (data not shown), but they are not completely synchronized as, on the contrary, it occurs (in accordance to our theoretical results) to nodes 4 and 12 . In the rest of the network we have not noticed any special behavior.

Finally, we show as a counterexample that a strategy which only considers a controller consisting in a single link between the two nodes 4 and 12 is not effective for synchronization. This corresponds to a network formed by the links denoted as continuous lines in Fig. 1 and a further link (not shown) between nodes 4 and 12 (in this case $\mathcal{L}^{\prime}$ would include a single link, i.e. $(4,12)$, with a weight as in the rest of the network, $\sigma=2$ ). Fig. 4 indicates that we do not observe synchronization between the two nodes. Therefore, directly connecting the two units with a link is an efficient synchronization strategy only if the two units are not embedded into a network.

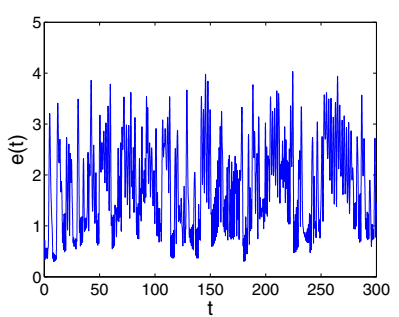

(a) (b)

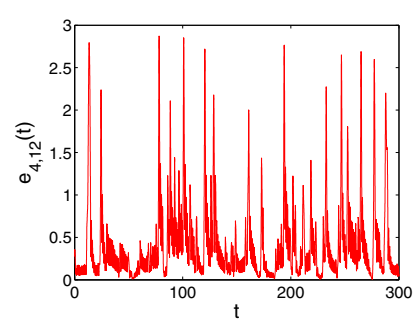

Fig. 4. Behavior of the dynamical network of Fig. 1 (continuous lines) when the controller consists of a single direct link between nodes 4 and 12: (a) global synchronization error $e(t)$; (b) synchronization error $e_{4,12}(t)$ between nodes 4 and 12. The introduction of a direct link between nodes 4 and 12 is not able to induce synchronization in them.

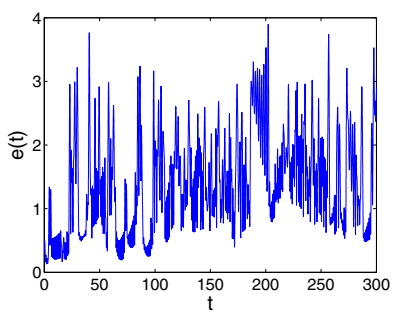

(a)

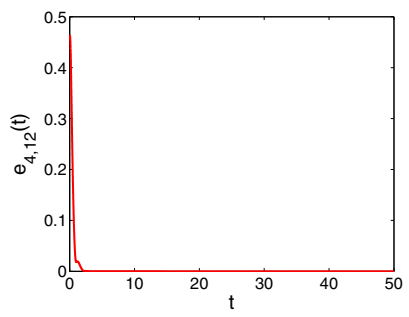

(b)
Fig. 5. Behavior of the dynamical network of Fig. 1 with heterogeneous units in presence of control (30): (a) global synchronization error $e(t)$; (b) synchronization error $e_{4,12}(t)$ between nodes 4 and 12. Control enables synchronization between the two target nodes, 4 and 12 , while the rest of the network remains not synchronized.

Example 2. In this example, we illustrate the case of a dynamical network with heterogeneous dynamics. In particular, we study a network with the same topology as in Example 1, but we now introduce heterogeneity in the oscillators through the parameter $\alpha$, which is now made node-dependent, i.e., $\alpha_{i}$. In particular, we set $\alpha_{i}=8.4$ for nodes $1,3,8,10,11,19$, and 20 , and $\alpha_{i}=10$ for all the other units. In this way, the isolated dynamics is different for the nodes with $\alpha_{i}=8.4$, displaying a single-scroll chaotic attractor, and for the others, displaying a double-scroll chaotic attractor. The other parameters and the goal of the control are the same as in Example 1. Note that the two nodes to synchronize have the same dynamics.

Fig. 5 shows the results when the distributed control (30) is applied. Nodes 4 and 12 are synchronized, while the rest of the network is not.

We note that, in both examples, the dynamical network achieves a form of synchronization which is known as remote [40]-[42]. In fact, the nodes in $\mathcal{S}_{n_{2}}$ synchronize even if they are not directly connected.

\section{CONCLUSION}

In this technical note we have studied the problem of controlling synchronization of an arbitrary set of dynamical units embedded in a networked system, independently from the states of the remaining units. We have assumed that the coupling gain is not controllable, but fixed, and we have devised a distributed control method to solve the problem. The 
proposed control relies on the creation of appropriate network symmetries through an additional layer of links, that ensures the stability of the induced synchronization manifold if the overall degree of the nodes to synchronize is made larger than a critical value, which is inversely proportional to the coupling coefficient. Quite interestingly, we have found that it is not important to which nodes the units to synchronize are connected, but only their number, a finding that paves the way to further optimization of the control strategy. The results presented in this work are formulated as a sufficient condition for a system of diffusively coupled identical dynamical units, and are obtained by using state transformations determined by network symmetry considerations and by Lyapunov functions. The extension to the case of a heterogeneous system of dynamical units has been also considered, showing that the important condition is that only the units to synchronize must have the same dynamics.

\section{REFERENCES}

[1] S. Strogatz, Sync: The emerging science of spontaneous order. Penguin UK, 2004

[2] A. Arenas, A. Díaz-Guilera, J. Kurths, Y. Moreno, and C. Zhou, "Synchronization in complex networks," Phys. Rep., vol. 469, no. 3, pp. 93-153, 2008

[3] Z. Li and G. Chen, "Global synchronization and asymptotic stability of complex dynamical networks," IEEE Trans. Circuits Syst. II, vol. 53, no. 1 , pp. $28-33,2006$.

[4] L. M. Pecora and T. L. Carroll, "Master stability functions for synchronized coupled systems," Phys. Rev. Lett., vol. 80, no. 10, p. 2109, 1998.

[5] G. Russo and M. Di Bernardo, "Contraction theory and master stability function: linking two approaches to study synchronization of complex networks," IEEE Trans. Circuits Syst. II, vol. 56, no. 2, pp. 177-181, 2009.

[6] V. N. Belykh, I. V. Belykh, and E. Mosekilde, "Cluster synchronization modes in an ensemble of coupled chaotic oscillators," Phys. Rev. E, vol. 63 , no. 3, p. 036216, 2001.

[7] M. J. Panaggio and D. M. Abrams, "Chimera states: coexistence of coherence and incoherence in networks of coupled oscillators," Nonlinearity, vol. 28, no. 3, p. R67, 2015.

[8] N. C. Rattenborg, C. Amlaner, and S. Lima, "Behavioral, neurophysiological and evolutionary perspectives on unihemispheric sleep," Neurosci. Biobehav. Rev., vol. 24, no. 8, pp. 817-842, 2000.

[9] I. J. Balaguer, Q. Lei, S. Yang, U. Supatti, and F. Z. Peng, "Control for grid-connected and intentional islanding operations of distributed power generation," IEEE Trans. Ind. Electron., vol. 58, no. 1, pp. 147-157, 2011.

[10] W. Yu, P. DeLellis, G. Chen, M. Di Bernardo, and J. Kurths, "Distributed adaptive control of synchronization in complex networks," IEEE Trans. Autom. Control, vol. 57, no. 8, pp. 2153-2158, 2012.

[11] R. Carli and S. Zampieri, "Network clock synchronization based on the second-order linear consensus algorithm," IEEE Trans. Autom. Control, vol. 59, no. 2, pp. 409-422, 2014.

[12] X. F. Wang and G. Chen, "Pinning control of scale-free dynamical networks," Physica A: Statistical Mechanics and its Applications, vol. 310, no. 3, pp. 521-531, 2002.

[13] X. Li, X. Wang, and G. Chen, "Pinning a complex dynamical network to its equilibrium," IEEE Trans. Circuits Syst. I, vol. 51, no. 10, pp. 2074-2087, 2004

[14] F. Sorrentino, M. di Bernardo, F. Garofalo, and G. Chen, "Controllability of complex networks via pinning," Phys. Rev. E, vol. 75, no. 4, p. 046103, 2007.

[15] M. Porfiri and M. Di Bernardo, "Criteria for global pinningcontrollability of complex networks," Automatica, vol. 44, no. 12, pp. 3100-3106, 2008.

[16] W. Yu, G. Chen, and J. Lü, "On pinning synchronization of complex dynamical networks," Automatica, vol. 45, no. 2, pp. 429-435, 2009.

[17] X. Liu and T. Chen, "Synchronization of complex networks via aperiodically intermittent pinning control," IEEE Trans. Autom. Control, vol. 60, no. 12 , pp. 3316-3321, 2015
[18] W. Wu, W. Zhou, and T. Chen, "Cluster synchronization of linearly coupled complex networks under pinning control," IEEE Trans. Circuits Syst. I, vol. 56, no. 4, pp. 829-839, 2009.

[19] H. Su, Z. Rong, M. Z. Chen, X. Wang, G. Chen, and H. Wang, "Decentralized adaptive pinning control for cluster synchronization of complex dynamical networks," IEEE Trans. Cybern., vol. 43, no. 1, pp. 394-399, 2013.

[20] C. B. Yu, J. Qin, and H. Gao, "Cluster synchronization in directed networks of partial-state coupled linear systems under pinning control," Automatica, vol. 50, no. 9, pp. 2341-2349, 2014.

[21] J. Sieber, E. Omelchenko, and M. Wolfrum, "Controlling unstable chaos: stabilizing chimera states by feedback," Phys. Rev. Lett., vol. 112, no. 5, p. $054102,2014$.

[22] I. Omelchenko, E. Omelchenko, A. Zakharova, M. Wolfrum, and E. Schöll, "Tweezers for chimeras in small networks," Phys. Rev. Lett., vol. 116, no. 11, p. 114101, 2016.

[23] L. V. Gambuzza and M. Frasca, "Pinning control of chimera states," Phys. Rev. E, vol. 94, no. 2, p. 022306, 2016.

[24] X. Liu and T. Chen, "Cluster synchronization in directed networks via intermittent pinning control," IEEE Trans. Neural Netw., vol. 22, no. 7 , pp. 1009-1020, 2011.

[25] X. Liu and S. Li, "Cluster synchronization for linearly coupled nonidentical systems with delays via aperiodically intermittent pinning control," IEEE Access, vol. 5, pp. 4179-4189, 2017.

[26] W. Lin, H. Fan, Y. Wang, H. Ying, and X. Wang, "Controlling synchronous patterns in complex networks," Phys. Rev. E, vol. 93, no. 4, p. $042209,2016$.

[27] D. Fiore, G. Russo, and M. di Bernardo, "Exploiting nodes symmetries to control synchronization and consensus patterns in multiagent systems," IEEE Control Syst. Lett., vol. 1, no. 2, pp. 364-369, 2017.

[28] S. Boccaletti, J. Kurths, G. Osipov, D. Valladares, and C. Zhou, "The synchronization of chaotic systems," Phys. Rep., vol. 366, no. 1, pp. 1-101, 2002.

[29] E. Estrada, The structure of complex networks: theory and applications. Oxford University Press, 2012.

[30] V. Latora, V. Nicosia, and G. Russo, Complex Networks: Principles, Methods and Applications. Cambridge University Press, 2017.

[31] V. Heine, Group theory in quantum mechanics: an introduction to its present usage. Courier Corporation, 2007.

[32] R. A. Horn and C. R. Johnson, Matrix analysis. Cambridge university press, 2012

[33] L. M. Pecora, F. Sorrentino, A. M. Hagerstrom, T. E. Murphy, and R. Roy, "Cluster synchronization and isolated desynchronization in complex networks with symmetries," Nat. Commun., vol. 5, p. 4079, 2014.

[34] C. Fu, W. Lin, L. Huang, and X. Wang, "Synchronization transition in networked chaotic oscillators: The viewpoint from partial synchronization," Phys. Rev. E, vol. 89, no. 5, p. 052908, 2014.

[35] J. Heagy, T. Carroll, and L. Pecora, "Synchronous chaos in coupled oscillator systems," Phys. Rev. E, vol. 50, no. 3, p. 1874, 1994.

[36] V. Andrieu, B. Jayawardhana, and L. Praly, "Transverse exponential stability and applications," IEEE Trans. Autom. Control, vol. 61, no. 11, pp. 3396-3411, 2016

[37] X. F. Wang and G. Chen, "Synchronization in scale-free dynamical networks: robustness and fragility," IEEE Trans. Circuits Syst. I, vol. 49 , no. 1, pp. 54-62, 2002.

[38] J. Lu, X. Yu, G. Chen, and D. Cheng, "Characterizing the synchronizability of small-world dynamical networks," IEEE Trans. Circuits Syst. $I$, vol. 51, no. 4, pp. 787-796, 2004.

[39] L. Fortuna, M. Frasca, and M. G. Xibilia, Chua's circuit implementations: yesterday, today and tomorrow. World Scientific, 2009, vol. 65.

[40] L. V. Gambuzza, A. Cardillo, A. Fiasconaro, L. Fortuna, J. GómezGardenes, and M. Frasca, "Analysis of remote synchronization in complex networks," Chaos, vol. 23, no. 4, p. 043103, 2013

[41] V. Nicosia, M. Valencia, M. Chavez, A. Díaz-Guilera, and V. Latora, "Remote synchronization reveals network symmetries and functional modules," Phys. Rev. Lett., vol. 110, no. 17, p. 174102, 2013.

[42] L. Zhang, A. E. Motter, and T. Nishikawa, "Incoherence-mediated 2017. 